\title{
A Hybrid WDM/TDM PON Architecture Using Wavelength Selective Switches
}

\author{
G. Das*, B. Lannoo*, D. Colle*, M. Pickavet*, P. Demeester* \\ * Department of Information Technology(INTEC), Ghent University-IBBT, Belgium, goutam.das@intec.ugent.be
}

\begin{abstract}
In this paper we propose some new hybrid WDM/TDM PON architectures that use wavelength selective switches at the remote node to improve flexibility, data security and power budget. We compare it with the existing WDM/TDM PONs in terms of cost and power budget.
\end{abstract}

\section{INTRODUCTION}

Time division multiplexing (TDM) passive optical networks (PONs), like Ethernet PON (EPON) and Gigabit PON (GPON), are now widely accepted as access network solution to distribute reasonably high bandwidths to the customers through a fiber infrastructure. However, due to the advent of bandwidth extensive services like high-definition video or interactive gaming, wavelength division multiplexing (WDM) PONs are introduced to increase capacity for each individual user. As these pure WDM PONs tend to waste bandwidth due to the lack of flexibility, different hybrid WDM/TDM PON architectures are proposed in the literature. One of the main flavors is wavelength broadcast and select (WBS) PON architecture that uses passive power splitters to broadcast all the wavelengths to all the users, and leave it to the media access control (MAC) layer to schedule time slots as well as wavelengths for different users. It requires tunability at the optical network unit (ONU) at the customer premises. Another WDM/TDM PON architecture, the wavelength splitting (WS) PON, uses a combination of a wavelength splitter (e.g., arrayed waveguide grating, AWG) and a power splitter to share each wavelength among multiple ONUs using TDM. It requires low-cost fixed transceivers at the ONU, however, the ONU becomes colored. Though this architecture significantly improves the power budget, it reduces the overall flexibility available in the WBS PON. The other architecture proposed in the literature is the wavelength routed (WR) PON, which uses a combination of power splitters and optical switches to switch any wavelength to any TDM PON [1],[2]. The WR PON significantly improves upon the data security compared to the WBS PON while keeping the flexibility of dynamically switching wavelengths from one TDM PON to another (contrary to the fixed wavelength allocation scheme of the WS PON). However, the WR PON introduces active equipment in the remote node and the combination of flexibility and security comes at the expense of power budget and cost [3].

In this paper, we propose a novel scheme to incorporate the good points of all three solutions mentioned above and to avoid the hind side of each. In particular, we propose to use wavelength selective switches (WSS) at the remote node (RN). This might increase the overall cost of the proposed architecture due to the expensive WSS as well as the requirement for power provisioning in presence of active components at the remote node. However, we will prove from our techno-economic analysis that the introduction of WSS in the RN does not affect the cost per customer significantly due to the higher reach, wavelength sharing and added flexibility. In the next section, we provide a brief description of the general hybrid WDM/TDM PON architecture before introducing the novel WSS PON proposal.

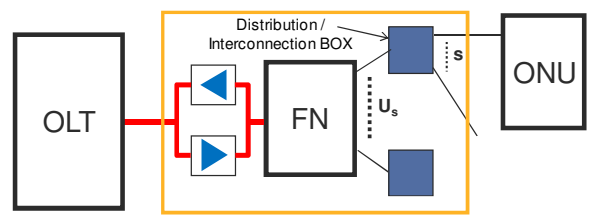

Fig. 1: General hybrid WDM/TDM PON architecture

\section{HYBRID TDM/WDM PON ARCHITECTURES}

The hybrid WDM/TDM PON using WSS, that we propose, has the general architecture as shown in Fig. 1 (introduced in [3]). The optical line terminal (OLT) has $N_{u}$ uplink (UL) and $N_{d}$ downlink (DL) line cards with respective fixed optical receiver and transmitter banks. Our RN is assumed to have two stages: stage one is the flexible node (FN) with WSS (shared by all users) and stage two has $U_{s}$ passive splitters for $U_{s}$ TDM PONs. Each TDM PON has $s$ ONUs.

For the optical network unit (ONU), two different types are shown in Fig. 2, and they were already discussed in [3].
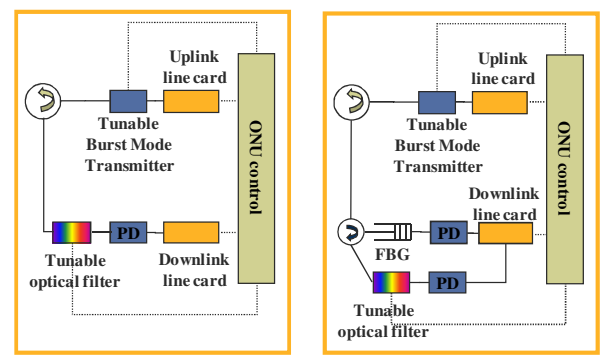

Fig. 2: Architecture of ONU a (left) and ONU b (right)

ONU type a (ONUa) contains a pair of transceivers for UL and DL data transmission. On the contrary, ONU type $b$ (ONUb) contains a tunable burst mode transmitter for UL data transmission and two photo detectors (PDs) (variable and fixed wavelength) for DL data transmission. The use of both a tunable and fixed receiver (Rx) improves the dynamic bandwidth allocation (DBA) significantly and simplifies the 


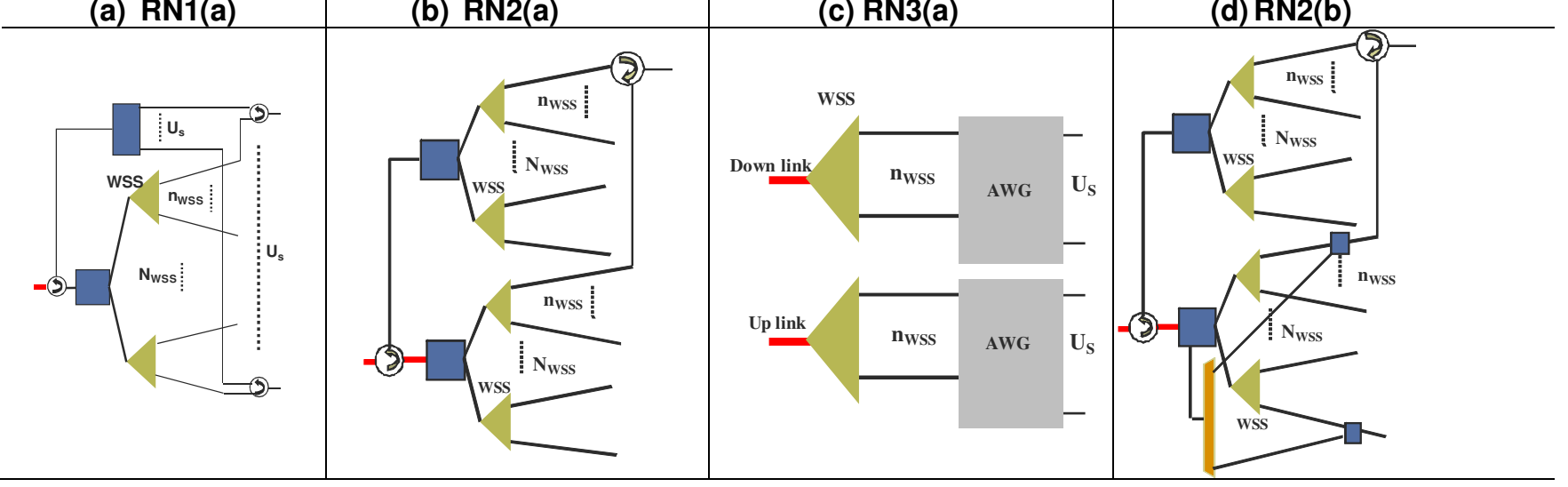

Fig. 3: Overview of remote node variants using WSS

MAC protocol to handle DBA. For further details of the operation of the MAC protocol, we refer to [1].

In the next section, we describe several variants of the flexible node architecture using WSS.

\section{WSS REMOTE NODE VARIATIONS}

WSS [4] are generally implemented in micro-electromechanical systems (MEMS) that provide low insertion loss wavelength switching capabilities. Off-the-shelf WSS can have the functionality of $1 \times 2,1 \times 4$ or $1 \times 8$ switching. They are available with both $100 \mathrm{GHz}$ and $50 \mathrm{GHz}$ channel spacing, i.e. with 48 and 96 wavelengths channels, respectively. When it is used as reconfigurable optical demultiplexer, a WSS can steer each optical channel present on its common input port towards one of its output ports. On the other hand, in the reverse direction it can be used as wavelength blocking device, where it can block some of the wavelengths from each of the ports to enter to the common port. However, it can be configured in a manner that it will allow all the possible wavelengths from each of the output ports to enter into the common port. Using WSS as the building block, three RN types are proposed (Fig. 3 ), each with two different variants for ONUa and ONUb, respectively.

\section{- RN type 1 [RN1(a)]:}

Fig. 3 (a) depicts the hybrid WDM/TDM PON architecture using WSS in the RN (and is targeted for ONUa). This architecture only uses WSS for the DL direction, where $N_{\text {WSS }}$ WSS are used. Each of the WSS is assumed to have $n_{\text {WSS }}$ output ports. $N_{W S S}$ is chosen according to the following formula: $N_{W S S}=U_{s} / n_{W S S}$.

The UL data transmissions from different ONUs are combined through an $U_{s} \times 1$ combiner.

\section{Advantages:}

(1) The power budget in the DL direction is much better than for the WBS PON due to the low insertion loss of a WSS ( $3 \mathrm{~dB}$ for $100 \mathrm{GHz}$ WSS). (2) A WSS facilitates the wavelength switching capabilities and therefore has more flexibility compared to the WS PON.

\section{Disadvantages:}

(1) A WSS is a comparatively costly device. However, as it can resolve the power budget as well as DBA issues, it can eventually increase the possibility of adding more customers to the same RN to eventually reduce the cost for an individual customer due to WSS sharing. (2) A WSS cannot facilitate DL broadcasting for a particular wavelength which is possible in the WBS PON. However, RN1 can do limited broadcasting as the same wavelength can be multicast to $N_{W S S}$ different output ports of the RN provided they are attached to different WSS modules. In addition to that, a WSS can provide dynamic capability of steering one wavelength from an output port to another one if the users attached to the concerned output port do not require the service of that wavelength. (3) RN1 still suffers from high losses due to the UL power splitting.

- RN type 2 [RN2(a)]:

Fig. 3 (b) depicts the architecture with WSS connected to both UL and DL direction (and is targeted for ONUa). For each TDM PON, the RN uses two ports of two different WSS, one for UL and one for DL data stream. The UL and DL data streams are combined by a circulator as shown in Fig. 3 (b). In the UL direction, the WSS are configured in such a manner that they allow all the wavelengths from the incoming ports. The architecture of RN2 enjoys all the advantages and disadvantages of using WSS. However, it also has the following pros and cons compared to RN1.

\section{Advantages:}

The power budget in the UL is improved due to the replacement of the passive coupler.

\section{Disadvantages:}

The cost of the $\mathrm{RN}$ is increased as the new configuration requires double the number of WSS (as each of the TDM PONs occupies two ports of two different WSS instead of one port required in RN1).

\section{- RN type 3 [RN3(a)]:}

Fig. 3 (c) depicts a cost effective and power efficient WSS configuration (and is targeted for ONUa). It still provides a limited wavelength switching capability. Here, we use just one WSS for UL and DL data transmission, respectively, in combination with two cyclic AWGs. However, due to the 
presence of the AWGs, only a specified number of wavelengths can reach a particular TDM PON. It is a trivial exercise to show that the maximum number of wavelengths that can reach a particular TDM PON is equal to the number $n_{W S S}$. Therefore, we can increase the flexibility of the proposed architecture with increasing the number of ports per WSS. This brings a cost vs. flexibility tradeoff issue in the architecture design.

\section{Advantages:}

(1) The power budget in the DL as well as UL direction is much better than for the other two RNs as well as for the WBS PON. (2) The cost of RN3 is the minimum among all the RNs proposed in this paper using WSS.

\section{Disadvantages:}

RN3 is restrictive in terms of flexibility as due to the presence of the AWG, it cannot steer any wavelength to any TDM PON. However, the flexibility can be improved by increasing the number of ports per WSS.

\section{- RN type 1(b), 2(b), 3(b) [RN1(b) / RN2(b) / RN3(b)]:}

We need to mention that the presented architectures for RN1, RN2 and RN3 only can be used with ONUa at the user side. Hence we introduce architectures RN1(b), RN2(b) and RN3(b) to be used with ONUb. Fig. 3 (d) depicts an example of the modified architecture of RN2 to be used with ONUb. We have used an AWG to demultiplex all the DL wavelengths and added them to the appropriate DL stream with the help of a passive combiner $(2 \times 1)$ as shown in Fig. 3 (d). In this way we ensure that the fixed wavelength assigned to a particular TDM PON is always routed to it, in addition to another dynamically assigned wavelength. The only disadvantage is the cost of additional components and a slightly reduced power budget due to the additional splitting loss coming from the added combiner.

Similarly RN1(b) and RN3(b) can be realized to be suitable for ONUb using an extra AWG and a power splitter.

\section{POWER Budget \&TeChNO-ECONOMIC Evaluation}

In this section, we first calculate the power budget and the optical reach possible for the different WSS PON architectures. Table 1 provides the summary of power loss due to the optical components and the maximum optical reach possible for different user bases $(1024,512$ and 256 users per hybrid WDM/TDM PON). We assume a power penalty [5] of $2 \mathrm{~dB}$ and an erbium-doped fiber amplifier (EDFA) gain of $20 \mathrm{~dB}$ in both the UL and DL direction. It is evident from Table 1 that the WS PON is the best and the WBS and WR PON are the worst in terms of optical reach, whereas the WS PON has no flexibility in terms of wavelength sharing. On the contrary, hybrid PONs using WSS (e.g., RN2 and RN3 with ONUa) have a very satisfactory optical reach and a reasonable amount of flexibility which makes them an interesting candidate for many applications.

Next in Fig. 4, we compare the cost per subscriber of different architectures. We ignore the fiber installation costs and the operational expenditures as they remain the same for each architecture. We only consider the ONUa variants and only the WSS PON variants with RN2 and RN3 as they demonstrate the lowest optical power loss. The comparison shows that the shared costs for the WSS PON with RN2 and the WR PON are in the higher side. However, the per subscriber cost for the WSS PON with RN3, WBS PON and WS PON are in the acceptable zone. Therefore, considering all selection criteria like flexibility, data security, cost and reach, the WSS PON with RN3 clearly becomes the winner.

Table 1: Power budget for different WDM/TDM PON architectures

\begin{tabular}{|c|c|c|c|c|}
\hline PON architecture & $\begin{array}{l}\text { Power loss in } \\
\text { dB, excl. } \\
\text { fiber loss } \\
1024 \text { user }\end{array}$ & $\begin{array}{l}\text { Reach } \\
(\mathbf{k m}) \\
\mathbf{1 0 2 4} \\
\text { users }\end{array}$ & $\begin{array}{l}\text { Reach } \\
(\mathbf{k m}) \\
\mathbf{5 1 2} \\
\text { users }\end{array}$ & $\begin{array}{l}\text { Reach } \\
(\mathbf{k m}) \\
256 \\
\text { users }\end{array}$ \\
\hline WBS PON ONUa (UL) & 36.5 & \multirow{2}{*}{26} & \multirow{2}{*}{36} & \multirow{2}{*}{46} \\
\hline WBS PON ONUa (DL) & 39.5 & & & \\
\hline WR PON ONUa (UL) & 37.5 & \multirow{2}{*}{24} & \multirow{2}{*}{34} & \multirow{2}{*}{44} \\
\hline WR PON ONUa (DL) & 40 & & & \\
\hline WS PON ONUa (UL) & 28.5 & \multirow{2}{*}{58} & \multirow{2}{*}{68} & \multirow{2}{*}{78} \\
\hline WS PON ONUa (DL) & 31.5 & & & \\
\hline RN1 PON ONUa (UL) & 38 & \multirow{2}{*}{32} & \multirow{2}{*}{42} & \multirow{2}{*}{52} \\
\hline RN1 PON ONUa (DL) & 36 & & & \\
\hline RN2 PON ONUa (UL) & 36 & \multirow{2}{*}{40} & \multirow{2}{*}{50} & \multirow{2}{*}{60} \\
\hline RN2 PON ONUa (DL) & 36 & & & \\
\hline RN3 PON ONUa (UL) & 29 & \multirow{2}{*}{56} & \multirow{2}{*}{66} & \multirow{2}{*}{76} \\
\hline RN3 PON ONUa (DL) & 32 & & & \\
\hline
\end{tabular}

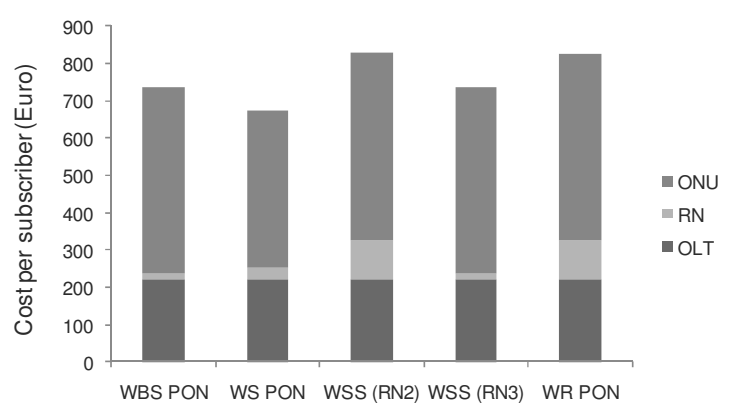

Fig. 4: Cost breakdown for different WDM/TDM PON architectures

\section{CONCLUSION}

We have proposed several variants of a hybrid WDM/ TDM PON using WSS and shown that it add some flexibility without compromising much of power budget and cost.

\section{ACKNOWLEDGMENT}

The research has received funding from the EC in the FP7 ICT-ALPHA project.

\section{REFERENCES}

[1] G.Das et al., Proc. ECOC'09, P6.28 (2009).

[2] T. Koonen, et al., Photonic Network Comm., vol. 3 (3), July 2001.

[3] B. Lannoo et al., Proc. ICTON'10, Mo.C4.3, Invited (2010).

[4] R. Jensen et al., Proc. OFC'08, OthA6 (2008).

[5] M. Takizawa et al., IEEE 802 Plenary Meeting, March 13-15, 2007. 